\title{
SNIPER SHOT PEGylation: TGase mediated site specific conjugation of PEG to proteins
}

\author{
Abhijeet Satwekar, Samanta Raboni, Barbara Spolaore, Nunzio Damiano and Angelo Fontana \\ CRIBI Biotechnology Center, University of Padua, Viale G. Colombo 3, 35121 Padua, Italy
}

\section{ABSTRACT}

The commercially avaliable recombinant protein drugs often cause immune reactions in the body which reduces its efficiency. Protein drugs can be PEGylated, (attachment of polyethylene glycol) to overcome this problem. PEGylation increases bioavaliability by reduced immune reactions and decreased renal clearnace [1]. So far the traditional approaches for PEGylation involve harsh reaction conditions which provides a heterogenous product (PEG attached randomly to different sites) along with the formation of several byproducts. Due to heterogenousity, the PEGylated protein drug faces challenge for the FDA approval. Therfore, there is an immense need to develop an approach which could generate a homogenous PEGylated protein drug.

The transglutaminase (TGase) is an enzyme which catalyses specifically the formation of a covalent bond (-CONH-) between the glutamine residue and the amine group of the lysine. This TGase reaction can be engineered by substituting the lysine with primary amines, which results into the formation of similar covalent bond between the glutamine and the primary amine [2]. We utilized this specificity of TGase by using primary PEG-amines for conjugation with the glutamine present in the model protein (apomyoglobin). The reaction conditions were optimised in order to get a mono conjugated PEGylated derivative. The site of conjugation was determined by affinity purification of the modified peptides and characterized by the ESI Q-TOF mass spectrometer. Therefore, we were able to develop a site specifically PEGylated apomyoglobin along with no byproducts, which eventually reduced the derivative purification steps as compared with the traditional PEGylation approaches. This strategy was further implemented on commercial pharmaceutical proteins.

Recombinant protein drugs have several limitations: 1. Susceptibility to degradation by proteases, 3. Propensity to generate immunogenic reactions.

Most promising solution $\Rightarrow$ Site specific covalent attachment of poly(ethylene glycol) (PEG) on the surface of proteins

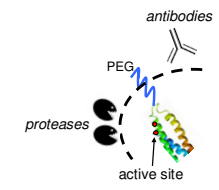

- Stability to proteolytic digestion Non-immunogenic,

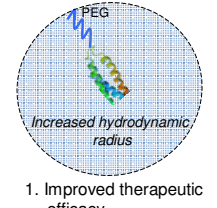

efficacy

2. Longer half-life than

native proteins

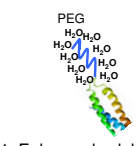

1. Enhanced solubility due 2.Non-aggregating,

\section{But, PEG conjugation at a specific site is CHALLENGING}

Therefore, We focused on the transglutaminase (TGase) approach for site specific conjugation of PEG on model protein (apomyoglobin).

Chemical approach Vs TGase approach

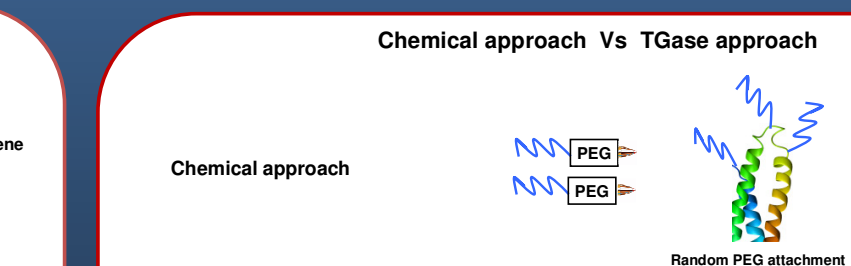

TGase approach

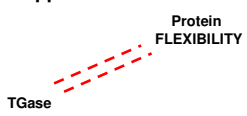

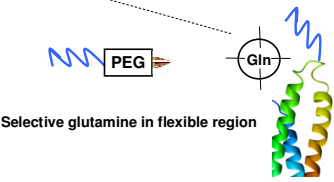

B] TGase mediated PEGylation

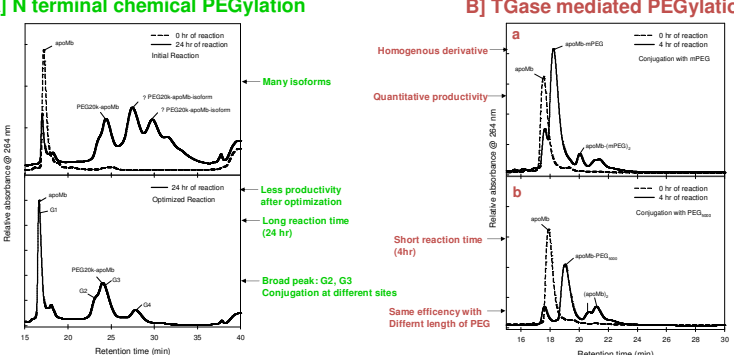

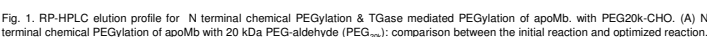

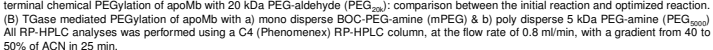

Characterization of site(s) of conjugation by ESI-Q-TOF mass spectrometer
Conjugation at the $\mathrm{N}$ terminal apomb[1-16] peptid
Conjugation at the level of Glutamine 91
Implementation on target pharmaceutical proteins

> The site specific PEGylation of a few valuable pharmaceutical proteins already in the clinical practice was achieved using the TGase approach.

$>$ The sites of conjugation by TGase were easily identified using biotinPEG-amine coupled to MS analysis.

$>$ Only one or few sites of derivatization were observed in the conjugated proteins (data not shown due to confidentiality of the work).

The glutamine residues that are derivatised are only those located in flexible regions of the proteins.
TGase attacks only the glutamine residues in the flexible region of protein.

Rapid characterization using Biotin-PEG-amine

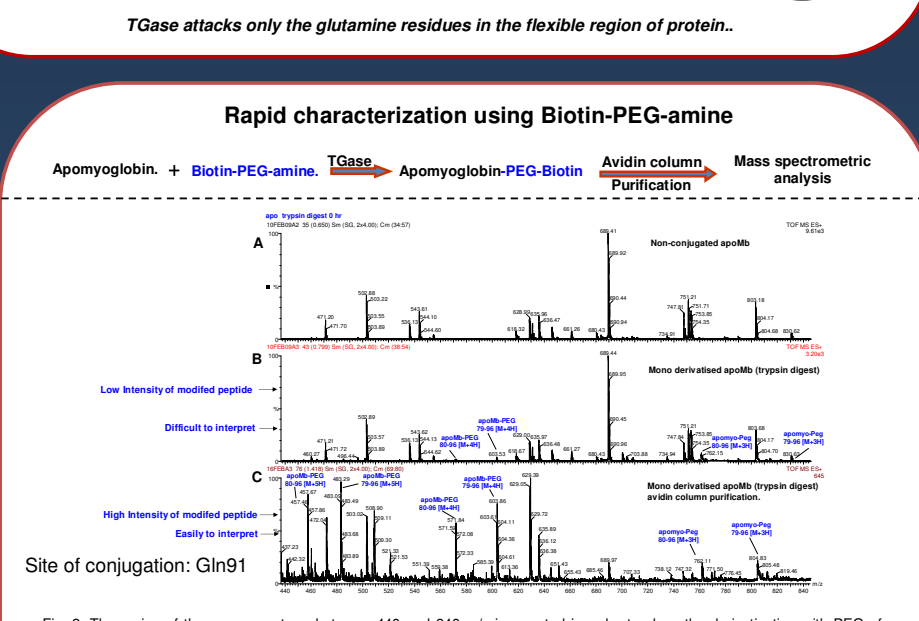

Fig. 2. The region of the mass spectrum between 440 and $840 \mathrm{~m} / \mathrm{z}$ is reported in order to show the derivatization with PEG of peptide 79-96 of apoMb. (A) Trypsin digest of non conjugated ap
digest of monoderivatised apoMb after avidin column purification.

\section{In spite of 6 glutamines in apomyoglobin sequence. $\Rightarrow$ \\ The conjugation occurred only on glutamine residue present in the flexible region}

\section{CONCLUSIONS}

- TGase mediated PEGylation using
Polydisperse PEG-NH
, \& biotin-PEG- $\mathrm{NH}_{2}$ - Use of (Biotin-PEG-NH
for affinity enrichment of Rapid accurate identification of the sites of TGase-mediated PEGylation modified peptides by mass spectrometry.

- Modified Gln residues of the investigated pharmaceutical proteins

located in disordered regions of the proteins

Hence, the sites of PEGylation in the target proteins could be predicted from the structure and dynamics as given by the X-ray or NMR data. 